\title{
The price of pain: the economics of chronic adolescent pain
}
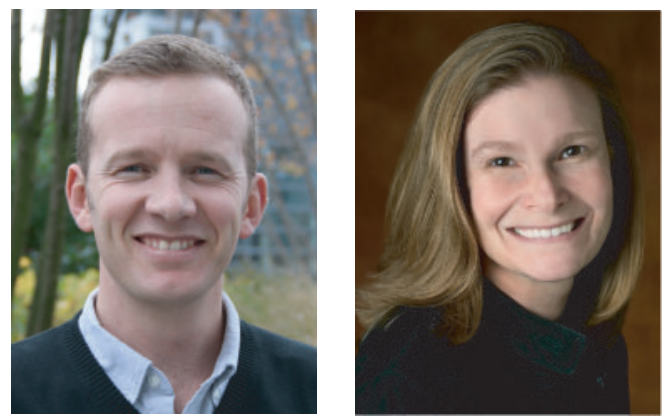

Cornelius B Groenewald ${ }^{*, 1} \&$ Tonya M Palermo'

Chronic pain is a distinct disease entity that affects adolescents' health in many tangible ways. Chronic pain is associated with anxiety and depression, fatigue, sleep disturbance, functional disability, and reduced quality of life [1-3]. Chronic pain also complicates other conditions: adolescents with anxiety disorders and depression are more likely to subsequently develop chronic pain and chronic pain is also a major cause of morbidity among adolescents with juvenile arthritis, sickle cell disease and those undergoing surgery [4-6]. Epidemiological studies estimate that up to a third of adolescents are affected by chronic pain [7]. Given the associated health consequences and high prevalence rate, it stands to reason that chronic adolescent pain may exert a significant economic burden on society. Surprisingly, this economic burden is largely unknown.

In contrast, it is well established that economic costs due to adult chronic pain are considerable. In the USA, the Institute of Medicine, a nonprofit, nongovernmental organization whose purpose is to provide national advice on health policy, recently commissioned a report aimed at estimating the state of pain treatment and research in the USA titled 'Relieving Pain in America' [8]. As part of this report, the Institute of Medicine estimated that chronic pain affected 100 million adults and was associated with up to US\$635 billion in healthcare costs and lost productivity in 2010. This is substantially more than the estimated costs for heart disease (309 billion), diabetes (188 billion) and cancer (243 billion) in America. However, even though the economic burden is staggering, funding for chronic pain is paltry. It is estimated that the National Institute of Health in the USA spends about 1.2\% of its budget on pain research [9]. This has clear implications: several groups, including the American Pain Society, have subsequently called for increased efforts to develop new therapies, optimize existing therapies, improve the understanding of pain and its mechanisms, and improve access to care in order to reduce the burden of adult chronic pain. Unfortunately, the Institute of Medicine did not include data on adolescent pain costs in their report.

\section{KEYWORDS}

- adolescent • economic

- healthcare use costs $\bullet$ pain

\section{"Given the associated health consequences and high prevalence rate, it stands to reason that chronic adolescent pain may exert a significant economic burden on society. Surprisingly, this economic burden is largely unknown."}




\section{"Studies of economic costs associated with adolescent chronic pain are urgently needed to inform government, health providers and the general public about the scope of the problem."}

\section{Current state of knowledge of adolescent pain costs}

Population-based studies have reported increased rates of health services use by children with chronic pain in Europe and Asia. In the Netherlands, Perquin et al. (2000) found high rates of physician consultation (57\%) and medication use $(39 \%)$ in a community sample of children with chronic pain [10]. Similarly, in Malaysia, Boey et al. (2001) found that $48.4 \%$ of children with abdominal pain consulted a medical practitioner over the previous year [11]. In a small single-site study from the USA, ToliverSokol et al. (2011) found that in a sample of youth seeking care for chronic pain in a multidisciplinary tertiary care pain clinic, $79.7 \%$ consulted healthcare providers and $72.9 \%$ received prescribed medications for pain over the previous 6 -month period [12]. The mean number of healthcare visits over 6 months incurred by participants in this study were 19 visits. While these studies confirmed high rates of healthcare use, they did not directly address associated economic costs. Currently, only two published studies have attempted to comprehensively estimate costs related to adolescent chronic pain, incorporating both direct (healthcare expenditures) and indirect (productivity losses due to parents' work absences) costs. In a small cohort of 52 adolescents, Sleed et al. (2005) estimated the mean annual cost per adolescent with chronic pain for the United Kingdom's National Health System at approximately $\$ 16,400$ (converted to 2012 US dollars) [13]. In another small cohort study involving 149 participants presenting to multiple tertiary care pain clinics, Groenewald et al. (2014) reported that adolescent chronic pain was associated with an annual average cost of $\$ 11,787$ (2012 US dollars) [14]. Both of these studies attempted to extrapolate their findings to national costs on adolescent pain, resulting in estimates of $\$ 9.5$ billion for the United Kingdom and $\$ 19.5$ billion for the USA annually (both converted to 2012 dollars). However, it is important to note that both these studies only enrolled adolescents with chronic pain problems severe enough to necessitate referral and evaluation in multidisciplinary tertiary care pain clinics, thereby likely overestimating costs. Furthermore, both studies had small samples, further limiting generalizability. Consequently, we have no reliable or solid estimates of the economic burden of chronic adolescent pain on society.

\section{Rationale for \& potential benefits of economic \& cost analyses}

Studies of economic costs associated with adolescent chronic pain are urgently needed to inform government, health providers and the general public about the scope of the problem. Data from such studies may help policy makers direct healthcare and research resources toward chronic pain. Additionally, cost analysis studies can help determine the potential cost savings for treating or preventing chronic pain. Cost analysis would also allow the comparison of costs between childhood diseases. Currently, attention-deficit hyperactivity disorder, asthma, and obesity are considered by both government and the lay public to be among the most costly pediatric diseases and funding for these conditions have become a priority. Directly comparing costs of pain to these priority conditions may give health decision-makers guidance on allocation of resources. A further benefit of cost analysis studies is that it provides important information for economic analysis such as costeffective, cost-benefit and cost-utility analyses. Economic analyses directly compare the relative costs and clinical outcomes of two or more alternative health treatments. It is unlikely for a new treatment to become widely adopted, unless it is shown to have at least comparable efficacy to other treatments and to save costs.

Methods of performing cost analysis: how should chronic pain costs be quantified?

While studies describing costs associated with chronic pain are urgently needed, it is important to not overestimate costs meant to urge decision makers into action. For example, extrapolating cost findings from adolescents presenting to tertiary care pain clinics may result in erroneously high national estimates since this represents only one end of the spectrum of adolescent chronic pain. While there are numerous reports describing how to conduct excellent cost analysis studies (see Larg et al. and Segel et al.), we will briefly discuss considerations in producing standardized and accurate cost analysis studies, using examples from the USA [15,16]:

- Specify the population being studied. There is currently a need to better estimate the economic burden of chronic pain on a societal level, both nationally and globally. In order to accomplish this goal, representative populations of adolescents with a wide spectrum of 
chronic pain problems and who receive care in different settings (e.g., primary care, specialty care) should be identified for study;

- Identify from whose perspective the costs are being considered. While several options are available, it is generally agreed that a societal perspective is the most appropriate. The societal perspective includes costs borne by all members of society, including the health system, family members and productivity losses to the economy. In contrast, cost analysis from a healthcare insurers' perspective may neglect to consider the impact on family's out of pocket costs;

- Comprehensively measure both direct and indirect costs. Direct costs are directly identifiable with a particular health service and are borne by the healthcare system and families such as hospital admissions, emergency department visits, outpatient visits, diagnostic tests, medications, other medical supplies and out of pocket costs. Indirect costs, on the other hand, measure the value of productivity lost due to pain, for example, parents taking time off work to care for a child in pain;

- Assign representative monetary values to healthcare use captured in studies. In the USA, one way to accomplish this is by applying unit cost data from the Medical Expenditure Panel Survey, which is the most complete source of data on healthcare use and costs for the civilian US population. Similar techniques exist for monetizing prescription medications use (using average wholesale prices from Thompson's Redbook) and human capital costs (average hourly wages) [14];
- Compare costs from studies performed in different years and different countries. This is surprisingly easy to do. To compare costs for the same country over several years, costs should be multiplied by the medical care component of the consumer price index. In the USA, these data can be found on the US Department of Labor website [14]. Similarly, costs between countries can be compared by converting to a standard monetary value based on current exchange rates.

\section{Conclusion}

Ample research has identified that adolescent chronic pain is a major cause of morbidity. Associated costs have not been well described, however, available data suggest that costs may mirror the enormous costs associated with adult chronic pain. Well-designed cost analysis studies are urgently needed to make pediatric chronic pain a priority condition, and to inform health policymakers on how best to allocate scarce funds for children and adolescents with chronic pain.

\section{Financial \& competing interests disclosure \\ This project was partially supported by National Institutes of Health K24HD060068 (PI:TM Palermo) and CB Groenewald was supported by National Institutes of Health Ruth L. Kirschstein National Research Service Award Institutional Research Training Grant T32GM086270 (PI:TM Palermo). The authors have no other relevant affiliations or financial involvement with any organization or entity with a financial interest in or financial conflict with the subject matter or materials discussed in the manuscript apart from those disclosed. \\ No writing assistance was utilized in the production of this manuscript.}

\section{References}

1 Luntamo T, Sourander A, Santalahti P, Aromaa M, Helenius H. Prevalence changes of pain, sleep problems and fatigue among 8-year-old children: years 1989, 1999, and 2005. J. Pediatr. Psychol. 37(3), 307-318 (2012).

2 Huguet A, Miro J. The severity of chronic pediatric pain: an epidemiological study. J. Pain 9(3), 226-236 (2008).

3 Tham SW, Holley AL, Zhou C, Clarke GN, Palermo TM. Longitudinal course and risk factors for fatigue in adolescents: the mediating role of sleep disturbances. J. Pediatr. Psychol. 38(10), 1070-1080 (2013).
4 Lewandowski Holley A, Law EF, Zhou C, Murphy L, Clarke G, Palermo TM.

Reciprocal longitudinal associations between pain and depressive symptoms in adolescents. Eur. J. Pain 17(7), 1058-1067 (2013).

5 Groenewald CB, Rabbitts JA, Schroeder DR, Harrison TE. Prevalence of moderate-severe pain in hospitalized children. Paediatr. Anaesth. 22(7), 661-668 (2012).

6 Weiss JE, Luca NJ, Boneparth A, Stinson J. Assessment and management of pain in juvenile idiopathic arthritis. Paediatr. Drugs 16(6), 473-481 (2014).

7 King S, Chambers CT, Huguet A et al. The epidemiology of chronic pain in children and adolescents revisited: a systematic review. Pain 152(12), 2729-2738 (2011).

8 Institute of Medicine. Relieving Pain in America: A Blueprint for Transforming Prevention, Care, Education, and Research. The National Academies Press, DC, USA (2011).

9 Gereau RWT, Sluka KA, Maixner W et al. A pain research agenda for the 21 st century. J. Pain 15(12), 1203-1214 (2014).

10 Perquin CW, Hazebroek-Kampschreur AA, Hunfeld JA, Van Suijlekom-Smit LW, Passchier J, Van Der Wouden JC. Chronic pain among children and adolescents: physician consultation and medication use. Clin. J. Pain 16(3), 229-235 (2000). 
11 Boey CC, Goh KL. Predictors of health-care consultation for recurrent abdominal pain among urban schoolchildren in Malaysia. J. Gastroenterol. Hepatol. 16(2), 154-159 (2001).

12 Toliver-Sokol M, Murray CB, Wilson AC, Lewandowski A, Palermo TM. Patterns and predictors of health service utilization in adolescents with pain: comparison between a community and a clinical pain sample. J. Pain 12(7), 747-755 (2011).
13 Sleed M, Eccleston C, Beecham J, Knapp M, Jordan A. The economic impact of chronic pain in adolescence: methodological considerations and a preliminary costs-ofillness study. Pain 119(1-3), 183-190 (2005).

14 Groenewald CB, Essner BS, Wright D, Fesinmeyer MD, Palermo TM. The economic costs of chronic pain among a cohort of treatment-seeking adolescents in the United States. J. Pain 15(9), 925-933 (2014).
15 Larg A, Moss JR. Cost-of-illness studies: a guide to critical evaluation.

Pharmacoeconomics 29(8), 653-671 (2011).

16 Segel J. Cost-of-illness studies--a primer. RTI-UNC Center of Excellence in Health Promotion Economics 1-39 (2006). www.rti.org/pubs/coi_primer.pdf 